\title{
Gravitomagnetic bending angle of light with finite-distance corrections in stationary axisymmetric spacetimes
}

\author{
Toshiaki Ono, Asahi Ishihara, and Hideki Asada \\ Graduate School of Science and Technology, \\ Hirosaki University, Aomori 036-8561, Japan
}

(Dated: November 9, 2017)

\begin{abstract}
By using the Gauss-Bonnet theorem, the bending angle of light in a static, spherically symmetric and asymptotically flat spacetime has been recently discussed, especially by taking account of the finite distance from a lens object to a light source and a receiver [Ishihara, Suzuki, Ono, Asada, Phys. Rev. D 95, 044017 (2017)]. We discuss a possible extension of the method of calculating the bending angle of light to stationary, axisymmetric and asymptotically flat spacetimes. For this purpose, we consider the light rays on the equatorial plane in the axisymmetric spacetime. We introduce a spatial metric to define the bending angle of light in the finite-distance situation. We show that the proposed bending angle of light is coordinate-invariant by using the Gauss-Bonnet theorem. The non-vanishing geodesic curvature of the photon orbit with the spatial metric is caused in gravitomagnetism, even though the light ray in the four-dimensional spacetime follows the null geodesic. Finally, we consider Kerr spacetime as an example in order to examine how the bending angle of light is computed by the present method. The finite-distance correction to the gravitomagnetic deflection angle due to the Sun's spin is around a pico-arcsecond level. The finitedistance corrections for Sgr A* also are estimated to be very small. Therefore, the gravitomagnetic finite-distance corrections for these objects are unlikely to be observed with present technology.
\end{abstract}

PACS numbers: 04.40.-b, 95.30.Sf, 98.62.Sb 


\section{INTRODUCTION}

Since the experimental confirmation of the theory of general relativity [1] succeeded in 1919 [2], a lot of calculations of the gravitational bending of light have been done not only for black holes [3-16] but also for other objects such as wormholes and gravitational monopoles [17-29]

Gibbons and Werner (2008) proposed an alternative way of deriving the deflection angle of light [30]. They assumed that the source and receiver are located at an asymptotic Minkowskian region and they used the Gauss-Bonnet theorem to a spatial domain described by the optical metric, for which a light ray is described as a spatial curve. Ishihara et al. have recently extended Gibbons and Werner's idea in order to investigate finite-distance corrections in the small deflection case (corresponding to a large impact parameter case) [31] and also in the strong deflection limit for which the photon orbits may have the winding number larger than unity [32]. In particular, the asymptotic receiver and source have not been assumed.

However, the earlier treatments [31, 32] are limited within the spherical symmetry. It is not clear whether the Gauss-Bonnet method with using the optical metric can be extended to axisymmetric cases or not. This is mostly because there can exist off-diagonal (timespace) components of the spacetime metric in an axisymmetric spacetime. The time-space components seem to make it unclear whether the optical metric can be constructed. After the gravitational lensing by a spinning object [33 35] and that by a relativistic binary [36] were discussed extensively by perturbative approaches such as the post-Newtonian approximation, Werner (2012) [37] proposed the use of the Kerr-Randers optical geometry on this issue [38]. To be more precise, he used the osculating Riemann approach in Finsler geometry in order to discuss the lensing by the Kerr black hole, for which the metric can be written in the Randers form. However, this approach requires that the endpoints (namely, the source and the receiver) of the photon orbit are in Euclidean space, for which angles can be easily defined. This requirement is mainly because jump angles at the vertices in the GaussBonnet theorem are problematic in the Finsler geometry. Namely, it is unlikely that the Finsler geometry can be used for computing the finite-distance corrections.

Therefore, the main purpose of the present paper is to extend the earlier formulation in

Refs. [31, 32], especially in order to examine finite-distance corrections to the deflection 
angle of light in the axisymmetric spacetime, for which the gravitational deflection of light may include gravitomagnetic effects (e.g. [33-36]). The geometrical setups in the present paper are not those in the optical geometry, in the sense that the photon orbit has a nonvanishing geodesic curvature, though the light ray in the four-dimensional spacetime obeys a null geodesic.

This paper is organized as follows. Section II discusses a possible extension to stationary and axisymmetric spacetimes. In particular, it is shown that the proposed definition of the deflection angle is coordinate-invariant by using the Gauss-Bonnet theorem. Section III uses the Kerr metric as a known example of the stationary and axisymmetric spacetimes in order to discuss how to compute the gravitational deflection angle of light by the proposed method. Section IV is devoted to conclusion. In Appendix A, the deflection angle of light is computed at the second order of the mass and the spin parameter in order to examine whether the deflection angle is in agreement with the known one. Throughout this paper, we use the unit of $G=c=1$, and the observer may be called the receiver in order to avoid a confusion between $r_{O}$ and $r_{0}$ by using $r_{R}$.

\section{EXTENSION TO AXISYMMETRIC SPACETIMES}

Henceforth, we assume a stationary and axisymmetric spacetime, for which we shall define the gravitational deflection angle of light by using the Gauss-Bonnet theorem: Suppose that $T$ is a two-dimensional orientable surface with boundaries $\partial T_{a}(a=1,2, \cdots, N)$ that are differentiable curves. See Figure 1. Let the jump angles between the curves be $\theta_{a}$ $(a=1,2, \cdots, N)$. Then, the Gauss-Bonnet theorem can be expressed as [41]

$$
\iint_{T} K d S+\sum_{a=1}^{N} \int_{\partial T_{a}} \kappa_{g} d \ell+\sum_{a=1}^{N} \theta_{a}=2 \pi
$$

where $K$ denotes the Gaussian curvature of the surface $T, d S$ is the area element of the surface, $\kappa_{g}$ means the geodesic curvature of $\partial T_{a}$, and $\ell$ is the line element along the boundary. The sign of the line element is chosen such that it is compatible with the orientation of the surface. 


\section{A. Stationary, axisymmetric spacetime}

We consider a stationary axisymmetric spacetime. The line element for this spacetime is [42-44]

$$
\begin{aligned}
d s^{2}= & g_{\mu \nu} d x^{\mu} d x^{\nu} \\
= & -A\left(y^{p}, y^{q}\right) d t^{2}-2 H\left(y^{p}, y^{q}\right) d t d \phi \\
& +F\left(y^{p}, y^{q}\right)\left(\gamma_{p q} d y^{p} d y^{q}\right)+D\left(y^{p}, y^{q}\right) d \phi^{2},
\end{aligned}
$$

where $\mu, \nu$ run from 0 to $3, p, q$ take 1 and $2, t$ and $\phi$ coordinates are associated with the Killing vectors, and $\gamma_{p q}$ is a two-dimensional symmetric tensor. It is more convenient to reexpress this metric into a form in which $\gamma_{p q}$ is diagonalized. The present paper prefers the polar coordinates rather than the cylindrical ones, because the Kerr metric in the polar coordinates is considered in Section III. In the polar coordinates, Eq. (2) becomes [45]

$$
\begin{aligned}
d s^{2}= & -A(r, \theta) d t^{2}-2 H(r, \theta) d t d \phi \\
& +B(r, \theta) d r^{2}+C(r, \theta) d \theta^{2}+D(r, \theta) d \phi^{2} .
\end{aligned}
$$

The null condition $d s^{2}=0$ is solved for $d t$ as [35]

$$
d t=\sqrt{\gamma_{i j} d x^{i} d x^{j}}+\beta_{i} d x^{i}
$$

where $i, j$ run from 1 to $3, \gamma_{i j}$ and $\beta_{i}$ are defined as

$$
\begin{aligned}
\gamma_{i j} d x^{i} d x^{j} & \equiv \frac{B(r, \theta)}{A(r, \theta)} d r^{2}+\frac{C(r, \theta)}{A(r, \theta)} d \theta^{2}+\frac{A(r, \theta) D(r, \theta)+H^{2}(r, \theta)}{A^{2}(r, \theta)} d \phi^{2}, \\
\beta_{i} d x^{i} & \equiv-\frac{H(r, \theta)}{A(r, \theta)} d \phi .
\end{aligned}
$$

This spatial metric $\gamma_{i j}\left(\neq g_{i j}\right)$ may define the arc length $(\ell)$ along the light ray as

$$
d \ell^{2} \equiv \gamma_{i j} d x^{i} d x^{j}
$$

for which $\gamma^{i j}$ is defined by $\gamma^{i j} \gamma_{j k}=\delta^{i}{ }_{k}$. Note that $\ell$ defined in this way is an affine parameter along the light ray. See e.g. Appendix of Ref. [35] for the proof on the affine parameter [46].

$\gamma_{i j}$ defines a 3-dimensional Riemannian space ${ }^{(3)} M$ in which the motion of the photon is described as a motion in a spatial curve. The unit tangential vector along the spatial curve is defined as

$$
e^{i} \equiv \frac{d x^{i}}{d \ell}
$$


The light ray follows the Fermat's principle [18]. By using the variational principle, this gives the equation for the light ray as [35]

$$
e^{i}{ }_{\mid k} e^{k}=a^{i}
$$

where | denotes the covariant derivative with $\gamma_{i j}$ and $a^{i}$ is defined as

$$
a^{i} \equiv \gamma^{i j}\left(\beta_{k \mid j}-\beta_{j \mid k}\right) e^{k}
$$

Here,

$$
e^{i}{ }_{\mid k} e^{k}=\frac{d e^{i}}{d \ell}+{ }^{(3)} \Gamma_{j k}^{i} e^{j} e^{k}
$$

where ${ }^{(3)} \Gamma^{i}{ }_{j k}$ denotes the Christoffel symbol associated with $\gamma_{i j}$.

The vector $a^{i}$ is the spatial vector that means the acceleration originated from $\beta_{i}$. In particular, $a^{i}$ is caused in gravitomagnetism as discussed below in more detail. This has an analogy as the acceleration by the Lorentz force $\propto \vec{v} \times\left(\vec{\nabla} \times \overrightarrow{A_{m}}\right)$ in electromagnetism, where $\vec{A}_{m}$ denotes the magnetic vector potential.

We should note that $\gamma_{i j}$ is not an induced metric. As a result, the photon orbit can deviate from a geodesic in ${ }^{(3)} M$ with $\gamma_{i j}$ if $\beta_{i} \neq 0$, even though the light ray in the four-dimensional spacetime follows the null geodesic.

For a stationary and spherically symmetric spacetime, one can always find a set of suitable coordinates such that $g_{0 i}$ can vanish to lead to $a^{i}=0$. In this case, the photon orbit becomes a spatial geodesic curve in ${ }^{(3)} M$.

The present paper discusses an extension to axisymmetric cases, which allow $g_{0 i} \neq 0$. Therefore, we have to take account of non-zero $\kappa_{g}$ along the photon orbit in the GaussBonnet theorem. This non-vanishing $\kappa_{g}$ of the photon orbit makes a crucial difference from the previous papers [31, 32]

\section{B. Geodesic curvature and equatorial plane}

Let us imagine a parameterized curve in a surface. The geodesic curvature of the parameterized curve is the surface-tangential component of acceleration (namely curvature) of the curve, while the normal curvature is the surface-normal component. The normal curvature 
has nothing to do with the present paper. The geodesic curvature can be defined in the vector form as (e.g. [47])

$$
\kappa_{g} \equiv \vec{T}^{\prime} \cdot(\vec{T} \times \vec{N})
$$

where we assume a parameterized curve with a parameter, $\vec{T}$ is the unit tangent vector for the curve by reparameterizing the curve using its arc length, $\vec{T}^{\prime}$ is its derivative with respect to the parameter, and $\vec{N}$ is the unit normal vector for the surface. In this paper, Eq. (12) can be rewritten in the tensor form as

$$
\kappa_{g}=\epsilon_{i j k} N^{i} a^{j} e^{k}
$$

where $\vec{T}$ and $\vec{T}^{\prime}$ correspond to $e^{k}$ and $a^{j}$, respectively. Here, the Levi-Civita tensor $\epsilon_{i j k}$ is defined by $\epsilon_{i j k} \equiv \sqrt{\gamma} \varepsilon_{i j k}$, where $\gamma \equiv \operatorname{det}\left(\gamma_{i j}\right)$, and $\varepsilon_{i j k}$ is the Levi-Civita symbol $\left(\varepsilon_{123}=1\right)$. In the present paper, the space is ${ }^{(3)} M$. Therefore, we use $\gamma_{i j}$ in the above definitions but not $g_{i j}$.

For a case of $a^{i} \neq 0$ due to $g_{0 i}$, there can exist a non-vanishing integral of the geodesic curvature along the light ray in the Gauss-Bonnet theorem by Eq. (11).

By substituting Eq. (10) into $a^{i}$ in Eq. (13), we obtain

$$
\kappa_{g}=-\epsilon^{i j k} N_{i} \beta_{j \mid k}
$$

where we use $\gamma_{i j} e^{i} e^{j}=1$.

Up to this point, the surface in ${ }^{(3)} M$ is not specified. Henceforth, we focus on the equatorial motion of the photon. We choose $\theta=\pi / 2$ as the equatorial plane. Then, the unit normal vector for the equatorial plane can be expressed as

$$
N_{p}=\frac{1}{\sqrt{\gamma^{\theta \theta}}} \delta_{p}^{\theta}
$$

where we choose the upward direction without loss of generality.

For the equatorial case, one can show

$$
\epsilon^{\theta p q} \beta_{q \mid p}=-\frac{1}{\sqrt{\gamma}} \beta_{\phi, r},
$$

where the comma denotes the partial derivative, we use $\epsilon^{\theta r \phi}=-1 / \sqrt{\gamma}$ and we note $\beta_{r, \phi}=0$ owing to the axisymmetry. By using Eqs. (15) and (16), an explicit form of $\kappa_{g}$ in Eq. (14) is obtained as

$$
\kappa_{g}=-\frac{1}{\sqrt{\gamma \gamma^{\theta \theta}}} \beta_{\phi, r} .
$$




\section{Impact parameter and the photon directions at the receiver and source}

We study the orbit equation on the equatorial plane with Eq. (3)). Associated with the two Killing vectors, there are the two constants of motion as

$$
\begin{aligned}
E & =A(r) \dot{t}+H(r) \dot{\phi}, \\
L & =D(r) \dot{\phi}-H(r) \dot{t},
\end{aligned}
$$

where the dot denotes the derivative with respect to the affine parameter.

As usual, we define the impact parameter as

$$
\begin{aligned}
b & \equiv \frac{L}{E} \\
= & \frac{-H(r) \dot{t}+D(r) \dot{\phi}}{A(r) \dot{t}+H(r) \dot{\phi}} \\
= & \frac{-H(r)+D(r) \frac{d \phi}{d t}}{A(r)+H(r) \frac{d \phi}{d t}}
\end{aligned}
$$

In terms of the impact parameter $b, d s^{2}=0$ leads to the orbit equation on the equatorial plane as

$$
\left(\frac{d r}{d \phi}\right)^{2}=\frac{A(r) D(r)+H^{2}(r)}{B(r)} \frac{D(r)-2 H(r) b-A(r) b^{2}}{[H(r)+A(r) b]^{2}},
$$

where we use Eq. (3). Let us introduce $u \equiv 1 / r$ to rewrite the orbit equation as

$$
\left(\frac{d u}{d \phi}\right)^{2}=F(u)
$$

where $F(u)$ is

$$
F(u)=\frac{u^{4}\left(A D+H^{2}\right)\left(D-2 H b-A b^{2}\right)}{B(H+A b)^{2}} .
$$

Finally, we examine the angles at the receiver and source positions. The unit tangent vector along the photon orbit in ${ }^{(3)} M$ is $e^{i}$. On the equatorial plane, its components are obtained as

$$
e^{i}=\frac{1}{\xi}\left(\frac{d r}{d \phi}, 0,1\right)
$$


Here, $\xi$ satisfies

$$
\frac{1}{\xi}=\frac{A(r)[H(r)+A(r) b]}{A(r) D(r)+H^{2}(r)},
$$

which can be derived from $\gamma_{i j} e^{i} e^{j}=1$ by using Eq. (21).

The unit radial vector in the equatorial plane is

$$
R^{i}=\left(\frac{1}{\sqrt{\gamma_{r r}}}, 0,0\right)
$$

where we choose the outgoing direction for a sign convention.

Therefore, we can define the angle measured from the outgoing radial direction by

$$
\begin{aligned}
\cos \Psi & \equiv \gamma_{i j} e^{i} R^{j} \\
& =\sqrt{\gamma_{r r}} \frac{A(r)[H(r)+A(r) b]}{A(r) D(r)+H^{2}(r)} \frac{d r}{d \phi},
\end{aligned}
$$

where Eqs. (24), (25) and (26) are used. This can be rewritten as

$$
\sin \Psi=\frac{H(r)+A(r) b}{\sqrt{A(r) D(r)+H^{2}(r)}},
$$

where we use Eq. (21). Note that $\sin \Psi$ by Eq. (28) is more convenient in practical calculations, because it needs only the local quantities, whereas $\cos \Psi$ by Eq. (27) needs the derivative as $d r / d \phi$.

\section{Deflection angle of light}

For the equatorial case in the axisymmetric spacetime, we define

$$
\alpha \equiv \Psi_{R}-\Psi_{S}+\phi_{R S} .
$$

This definition seems to rely on a choice of the angular coordinate $\phi$.

Let us consider a quadrilateral ${ }_{R}^{\infty} \square_{S}^{\infty}$, which consists of the spatial curve for the light ray, two outgoing radial lines from $\mathrm{R}$ and from $\mathrm{S}$ and a circular arc segment $C_{r}$ of coordinate radius $r_{C}\left(r_{C} \rightarrow \infty\right)$ centered at the lens which intersects the radial lines through the receiver or the source. See Figure 2 for the configuration such as the domain ${ }_{R}^{\infty} \square_{S}^{\infty}$. See also Ref. [32] for the case that the winding number is larger than unity. For the asymptotically flat spacetime, $\kappa_{g} \rightarrow 1 / r_{C}$ and $d \ell \rightarrow r_{C} d \phi$ as $r_{C} \rightarrow \infty$ (See e.g. [30]). Hence, $\int_{C_{r}} \kappa_{g} d \ell \rightarrow \phi_{R S}$. 
By using the Gauss-Bonnet theorem Eq. (11), Eq. (29) is rewritten as

$$
\alpha=-\iint_{\substack{\infty \\ \square_{S}^{\infty}}} K d S+\int_{S}^{R} \kappa_{g} d \ell,
$$

where $d \ell$ is positive for the prograde motion of the photon and it is negative for the retrograde motion. Eq. (30) shows that $\alpha$ is coordinate-invariant also for the axisymmetric case.

Up to this point, equations for gravitational fields are not specified. Therefore, the above discussion and results are not limited within the theory of general relativity (GR) but they are applicable to a certain class of modified gravity theories if the light ray in the fourdimensional spacetime obeys the null geodesic.

\section{APPLICATION TO THE KERR LENS}

\section{A. Kerr spacetime and $\gamma_{i j}$}

This section focuses on the Kerr spacetime as one of the most known examples with axisymmetry. The Boyer-Lindquist form of the Kerr metric is

$$
\begin{aligned}
d s^{2}= & -\left(1-\frac{2 M r}{\Sigma}\right) d t^{2}-\frac{4 a M r \sin ^{2} \theta}{\Sigma} d t d \phi \\
& +\frac{\Sigma}{\Delta} d r^{2}+\Sigma d \theta^{2}+\left(r^{2}+a^{2}+\frac{2 a^{2} M r \sin ^{2} \theta}{\Sigma}\right) \sin ^{2} \theta d \phi^{2}
\end{aligned}
$$

where $\Sigma$ and $\Delta$ are denoted as

$$
\begin{aligned}
& \Sigma \equiv r^{2}+a^{2} \cos ^{2} \theta, \\
& \Delta \equiv r^{2}-2 M r+a^{2} .
\end{aligned}
$$

By using Eqs. (5) and (6), one can see that $\gamma_{i j}$ and $\beta_{i}$ for the Kerr metric are given by

$$
\begin{aligned}
\gamma_{i j} d x^{i} d x^{j}= & \frac{\Sigma^{2}}{\Delta(\Sigma-2 M r)} d r^{2}+\frac{\Sigma^{2}}{(\Sigma-2 M r)} d \theta^{2} \\
& +\left(r^{2}+a^{2}+\frac{2 a^{2} M r \sin ^{2} \theta}{(\Sigma-2 M r)}\right) \frac{\Sigma \sin ^{2} \theta}{(\Sigma-2 M r)} d \phi^{2}, \\
\beta_{i} d x^{i}= & -\frac{2 a M r \sin ^{2} \theta}{(\Sigma-2 M r)} d \phi .
\end{aligned}
$$

Note that $\gamma_{i j}$ has no terms linear in the Kerr parameter $a$, because $g_{0 i} \propto H$ enters $\gamma_{i j}$ in a quadratic form through $g_{0 i} g_{0 j} \propto H^{2}$ as shown by Eq. (5).

In order to see what is $\kappa_{g}$ for the present case, we employ the weak field and slow rotation approximations, for which $M$ and $a$ can be used as book-keeping parameters. 


\section{B. Path integral of $\kappa_{g}$}

By substituting $\beta_{i}$ by Eq. (35) into Eq. (17), we obtain

$$
\begin{aligned}
\kappa_{g} & =-\frac{2 a M}{r^{2}(r-2 M)}\left(\frac{1-\frac{2 M}{r}+\frac{a^{2}}{r^{2}}}{1+\frac{a^{2}}{r^{2}}+\frac{2 a^{2} M}{r^{3}}}\right)^{1 / 2} \\
& =-\frac{2 a M}{r^{3}}+O\left(\frac{a M^{2}}{r^{4}}\right),
\end{aligned}
$$

where we use the weak field and slow rotation approximations in the last line and the terms of $a^{n} M(n \geq 2)$ vanish.

The path integral of $\kappa_{g}$ is computed as

$$
\begin{aligned}
\int_{S}^{R} \kappa_{g} d \ell & =-\int_{S}^{R}\left[\frac{2 a M}{r^{3}}+O\left(\frac{a M^{2}}{r^{4}}\right)\right] d \ell \\
& =-\frac{2 a M}{b^{2}} \int_{\phi_{S}}^{\phi_{R}} \cos \vartheta d \vartheta+O\left(\frac{a M^{2}}{b^{3}}\right) \\
& =-\frac{2 a M}{b^{2}}\left[\sqrt{1-b^{2} u_{R}^{2}}+\sqrt{1-b^{2} u_{S}^{2}}\right]+O\left(\frac{a M^{2}}{b^{3}}\right),
\end{aligned}
$$

where we assume the prograde case $d \ell>0$ that the orbital angular momentum of the photon is aligned with the spin of the black hole and we use a linear approximation of the photon orbit as $r=b / \cos \vartheta+O(M, a)$ and $\ell=b \tan \vartheta+O(M, a)$ in the second line. Note that, in the retrograde case, the sign of $d \ell$ is negative and thus the magnitude of the above path integral remains the same but the sign is opposite.

\section{C. $\phi_{R S}$ part}

The integral of the angular coordinate $\phi$ becomes

$$
\begin{aligned}
\phi_{R S} & =\int_{S}^{R} d \phi \\
& =2 \int_{0}^{u_{0}} \frac{1}{\sqrt{F(u)}} d u+\int_{u_{S}}^{0} \frac{1}{\sqrt{F(u)}} d u+\int_{u_{R}}^{0} \frac{1}{\sqrt{F(u)}} d u,
\end{aligned}
$$


where we use the orbit equation given by Eq. (22), By substituting Eq. (23) into $F(u)$ in Eq. (38), we obtain

$$
\begin{aligned}
\phi_{R S}= & \int_{u_{S}}^{u_{0}}\left(\frac{1}{\sqrt{u_{0}^{2}-u^{2}}}+M \frac{u_{0}^{3}-u^{3}}{\left(u_{0}^{2}-u^{2}\right)^{3 / 2}}-2 a M \frac{u_{0}^{3}\left(u_{0}-u\right)}{\left(u_{0}^{2}-u^{2}\right)^{3 / 2}}\right) d u \\
& +\int_{u_{R}}^{u_{0}}\left(\frac{1}{\sqrt{u_{0}^{2}-u^{2}}}+M \frac{u_{0}^{3}-u^{3}}{\left(u_{0}^{2}-u^{2}\right)^{3 / 2}}-2 a M \frac{u_{0}^{3}\left(u_{0}-u\right)}{\left(u_{0}^{2}-u^{2}\right)^{3 / 2}}\right) d u \\
& +O\left(M^{2}, a^{2}\right) \\
= & \left(\frac{\pi}{2}-\arcsin \left(\frac{u_{S}}{u_{0}}\right)+M \frac{\left(2 u_{0}+u_{S}\right) \sqrt{u_{0}^{2}-u_{S}^{2}}}{u_{0}+u_{S}}-2 a M \frac{u_{0}^{3} \sqrt{u_{0}^{2}-u_{S}^{2}}}{u_{0}^{2}+u_{0} u_{S}}\right) \\
& +\left(\frac{\pi}{2}-\arcsin \left(\frac{u_{R}}{u_{0}}\right)+M \frac{\left(2 u_{0}+u_{R}\right) \sqrt{u_{0}^{2}-u_{R}^{2}}}{u_{0}+u_{R}}-2 a M \frac{u_{0}^{3} \sqrt{u_{0}^{2}-u_{R}^{2}}}{u_{0}^{2}+u_{0} u_{R}}\right) \\
& +O\left(M^{2} u_{0}^{2}, a^{2} u_{0}^{2}\right)
\end{aligned}
$$

where we assume the prograde case. For the retrograde case, the sign of the term linear in $a$ becomes opposite.

Eq. (22) gives the relation between the impact parameter $b$ and the inverse of the closest approach $u_{0}$ as $b=u_{0}^{-1}+M-2 a M u_{0}+O\left(M^{2}, a^{2}\right)$ in the weak field and slow rotation approximations. By using this relation, $a M$ part of $\phi_{R S}$ in Eq. (39) can be rewritten in terms of $b$ as

$$
-\frac{2 a M}{b^{2}}\left[\frac{1}{\sqrt{1-b^{2} u_{S}^{2}}}+\frac{1}{\sqrt{1-b^{2} u_{R}^{2}}}\right] .
$$

See Eq. (32) of Ref. [31] for $M$ part of $\phi_{R S}$.

\section{D. $\Psi$ parts}

For the Kerr metric by Eq. (31), Eq. (28) becomes

$$
\sin \Psi=\frac{b}{r} \times \frac{1-\frac{2 M}{r}+\frac{2 a M}{b r}}{\sqrt{1-\frac{2 M}{r}+\frac{a^{2}}{r^{2}}}} .
$$

This is approximated as

$$
\sin \Psi=\frac{b}{r}\left(1-\frac{M}{r}+\frac{2 a M}{b r}\right)+O\left(\frac{M^{2}}{r^{2}}, \frac{a^{2}}{r^{2}}, \frac{a M^{2}}{r^{3}}\right) .
$$


By using this, we obtain

$$
\begin{aligned}
\Psi_{R}-\Psi_{S}= & \arcsin \left(b u_{R}\right)+\arcsin \left(b u_{S}\right)-\pi \\
& -\frac{M b u_{R}^{2}}{\sqrt{1-b^{2} u_{R}^{2}}}-\frac{M b u_{S}^{2}}{\sqrt{1-b^{2} u_{S}^{2}}} \\
& +\frac{2 a M u_{R}^{2}}{\sqrt{1-b^{2} u_{R}^{2}}}+\frac{2 a M u_{S}^{2}}{\sqrt{1-b^{2} u_{S}^{2}}} \\
& +O\left(M^{2} u_{R}^{2}, M^{2} u_{S}^{2}, a^{2} u_{R}^{2}, a^{2} u_{S}^{2}, a M^{2} u_{R}^{3}, a M^{2} u_{S}^{3}\right) .
\end{aligned}
$$

\section{E. Deflection angle of light in Kerr spacetime}

By substituting Eqs. (40) and (43) into Eq. (29), the deflection angle of light on the equatorial plane in the Kerr spacetime is obtained as

$$
\begin{aligned}
\alpha_{\text {prog }}= & \frac{2 M}{b}\left(\sqrt{1-b^{2} u_{S}^{2}}+\sqrt{1-b^{2} u_{R}^{2}}\right) \\
& -\frac{2 a M}{b^{2}}\left(\sqrt{1-b^{2} u_{R}^{2}}+\sqrt{1-b^{2} u_{S}^{2}}\right)+O\left(\frac{M^{2}}{b^{2}}\right),
\end{aligned}
$$

where we assume the prograde motion of light. For the retrograde case, it is

$$
\begin{aligned}
\alpha_{\text {retro }}= & \frac{2 M}{b}\left(\sqrt{1-b^{2} u_{S}^{2}}+\sqrt{1-b^{2} u_{R}^{2}}\right) \\
& +\frac{2 a M}{b^{2}}\left(\sqrt{1-b^{2} u_{R}^{2}}+\sqrt{1-b^{2} u_{S^{2}}}\right)+O\left(\frac{M^{2}}{b^{2}}\right) .
\end{aligned}
$$

Note that $a^{2}$ terms at the second order in the deflection angle cancel out. See Appendix A for more detail.

For both cases, we take the far limit as $u_{R} \rightarrow 0$ and $u_{S} \rightarrow 0$. Then, we obtain

$$
\begin{gathered}
\alpha_{\infty \text { prog }} \rightarrow \frac{4 M}{b}-\frac{4 a M}{b^{2}}+O\left(\frac{M^{2}}{b^{2}}\right), \\
\alpha_{\infty \text { retro }} \rightarrow \frac{4 M}{b}+\frac{4 a M}{b^{2}}+O\left(\frac{M^{2}}{b^{2}}\right),
\end{gathered}
$$

which show that Eqs. (44) and (45) recover the asymptotic deflection angles that are known in literature [4, 33, $\underline{34}$ ].

\section{F. Finite-distance corrections to the gravitomagnetic deflection angle of light}

The above calculations discuss the deflection angle of light due to the rotation of the lens

(its spin parameter $a$ ). In particular, we do not assume that the receiver and the source are 
located at the infinity. The finite-distance correction to the deflection angle of light, denoted as $\delta \alpha$, is the difference between the asymptotic deflection angle $\alpha_{\infty}$ and the deflection angle for the finite distance case. It is expressed as

$$
\delta \alpha \equiv \alpha-\alpha_{\infty}
$$

Eqs. (44) and (45) suggest the magnitude of the finite-distance correction to the gravitomagnetic deflection angle by the spin as

$$
\begin{aligned}
\left|\delta \alpha_{G M}\right| & \sim O\left(\frac{a M}{r_{S}^{2}}+\frac{a M}{r_{R}^{2}}\right) \\
& \sim O\left(\frac{J}{r_{S}^{2}}+\frac{J}{r_{R}^{2}}\right),
\end{aligned}
$$

where $J \equiv a M$ is the spin angular momentum of the lens and the subscript $G M$ denotes the gravitomagnetic part. As usual, we introduce the dimensionless spin parameter as $s \equiv a / M$. Hence, Eq. (49) is rewritten as

$$
\left|\delta \alpha_{G M}\right| \sim O\left(s\left(\frac{M}{r_{S}}\right)^{2}+s\left(\frac{M}{r_{R}}\right)^{2}\right) .
$$

This suggests that $\delta \alpha$ is comparable to the second post-Newtonian effect (multiplied by the dimensionless spin parameter). It is known that the second-order Schwarzschild contribution to $\alpha$ is $15 \pi M^{2} / 4 b^{2}$. This contribution can be found also by using the present method, especially by computing $\phi_{R S}$, where we use a relation between $b$ and $r_{0}$ in $M^{2}$. Please see Appendix A for detailed calculations at the second order of $M$ and $a$, especially the integrals of $K$ and $\kappa_{g}$ in the present formulation. See also the next subsection.

Note that $\delta \alpha$ at the leading order in the approximations does not depend on the impact parameter $b$. In fact, $\delta \alpha$ depends much weakly on $b$.

\section{G. Possible astronomical applications}

We discuss possible astronomical applications. First, we consider the Sun, where we ignore its higher multipole moments. The spin angular momentum of the Sun $J_{\odot}$ is $\sim$

$2 \times 10^{41} \mathrm{~m}^{2} \mathrm{~kg} \mathrm{~s}^{-1}$ [48]. Thus, $G J_{\odot} c^{-2} \sim 5 \times 10^{5} \mathrm{~m}^{2}$, which implies the dimensionless spin parameter as $s_{\odot} \sim 10^{-1}$. 
We assume that an observer at the Earth sees the light bending by the solar mass, while the source is practically at the asymptotic region. If the light ray passes near the solar surface, Eq. (50) implies that the finite-distance correction to this case is of the order of

$$
\begin{aligned}
\left|\delta \alpha_{G M}\right| & \sim O\left(\frac{J}{r_{R}^{2}}\right) \\
& \sim 10^{-12} \text { arcsec. } \times\left(\frac{J}{J_{\odot}}\right)\left(\frac{1 \mathrm{AU}}{r_{R}}\right)^{2},
\end{aligned}
$$

where $4 M_{\odot} / R_{\odot} \sim 1.75$ arcsec. $\sim 10^{-5}$ rad., and $R_{\odot}$ denotes the solar radius. This correction is around a pico-arcsecond level and thus it is unlikely to be observed with present technology [49, 50].

Please see Figure 3 for numerical calculations of the finite-distance correction due to the receiver location. The numerical results are consistent with the above order-of-magnitude estimation. The figure suggests that the dependence of $\delta \alpha$ on the impact parameter $b$ is very weak.

Next, we consider Sgr A* at the center of our Galaxy, which is expected as one of the most plausible candidates for the strong deflection of light. In this case, the receiver distance is much larger than the impact parameter of light, while a source star may be in the central region of our Galaxy.

For Sgr A*, Eq. (49) implies

$$
\begin{aligned}
\left|\delta \alpha_{G M}\right| & \sim s\left(\frac{M}{r_{S}}\right)^{2} \\
& \sim 10^{-7} \operatorname{arcsec} . \times\left(\frac{s}{0.1}\right)\left(\frac{M}{4 \times 10^{6} M_{\odot}}\right)^{2}\left(\frac{0.1 \mathrm{pc}}{r_{S}}\right)^{2},
\end{aligned}
$$

where we assume the mass of the central black hole as $M \sim 4 \times 10^{6} M_{\odot}$. This correction around a sub-microarcsecond level is unlikely to be measured with present technology.

Please see Figure 4 for numerical calculations of the finite-distance correction due to the source location. The numerical results are consistent with the above order-of-magnitude estimation. The figure shows that the dependence on the impact parameter $b$ is very weak. 


\section{H. Consistency of the present formulation}

Before closing this section, let us check the consistency of the above formulation. The Gaussian curvature is related with the 2-dimensional Riemann tensor as [37]

$$
\begin{aligned}
K & =\frac{{ }^{(3)} R_{r \phi r \phi}}{\gamma} \\
& =\frac{1}{\sqrt{\gamma}}\left[\frac{\partial}{\partial \phi}\left(\frac{\sqrt{\gamma}}{\gamma_{r r}}{ }^{(3)} \Gamma_{r r}^{\phi}\right)-\frac{\partial}{\partial r}\left(\frac{\sqrt{\gamma}}{\gamma_{r r}}{ }^{(3)} \Gamma_{r \phi}^{\phi}\right)\right],
\end{aligned}
$$

where ${ }^{(3)} \Gamma_{j k}^{i}$ and ${ }^{(3)} R_{a b c d}$ are associated with $\gamma_{i j}$. For the Kerr case, it becomes

$$
\begin{aligned}
K & =-\sqrt{\frac{A^{3}}{B\left(A D+H^{2}\right)}} \frac{\partial}{\partial r}\left[\frac{1}{2} \sqrt{\frac{A^{3}}{B\left(A D+H^{2}\right)}} \frac{\partial}{\partial r}\left(\frac{A D+H^{2}}{A^{2}}\right)\right] \\
& =-\frac{2 M}{r^{3}}+O\left(\frac{M^{2}}{r^{4}}, \frac{a^{2} M}{r^{5}}\right),
\end{aligned}
$$

where we use the weak field and slow rotation approximations in the last line. Note that $K$ has no terms linear in $a$. This is because $\gamma_{i j}$ has no terms linear $a$ as already mentioned. Furthermore, $a^{2}$ terms cancel out in $K$. See Appendix A for more detail.

In order to compute the surface integral of the Gaussian curvature in the Gauss-Bonnet theorem, we need know the integration domain, especially the photon orbit $S \rightarrow R$ for the present case. By straightforward calculations, the iterative solution of Eq. (22) for the Kerr case in the weak field and slow rotation approximations is obtained as

$$
\begin{aligned}
u= & \frac{1}{b} \sin \phi+\frac{M}{b^{2}}\left(1+\cos ^{2} \phi\right) \\
& -\frac{2 a M}{b^{3}}+O\left(\frac{M^{2}}{b^{3}}, \frac{a^{2}}{b^{3}}\right) .
\end{aligned}
$$

By using this, the surface integral of the Gaussian curvature is computed as

$$
\begin{aligned}
-\iint_{c_{R} \square_{S}^{\infty}} K d S & =\int_{r_{O E}}^{\infty} d r \int_{\phi_{S}}^{\phi_{R}} d \phi \frac{2 M}{r^{2}}+O\left(\frac{M^{2}}{b^{2}}, \frac{a M^{2}}{b^{3}}\right) \\
& =2 M \int_{\phi_{S}}^{\phi_{R}} d \phi \int_{0}^{\frac{1}{b} \sin \phi+\frac{M}{b^{2}}\left(1+\cos ^{2} \phi\right)-\frac{2 a M}{b^{3}}} d u+O\left(\frac{M^{2}}{b^{2}}, \frac{a M^{2}}{b^{3}}\right) \\
& =2 M \int_{\phi_{S}}^{\phi_{R}} d \phi[u]_{u=0}^{\frac{1}{b} \sin \phi+\frac{M}{b^{2}}\left(1+\cos ^{2} \phi\right)-\frac{2 a M}{b^{3}}}+O\left(\frac{M^{2}}{b^{2}}, \frac{a M^{2}}{b^{3}}\right) \\
& =\frac{2 M}{b} \int_{\phi_{S}}^{\phi_{R}} d \phi \sin \phi+O\left(\frac{M^{2}}{b^{2}}, \frac{a M^{2}}{b^{3}}\right) \\
& =\frac{2 M}{b}\left[\sqrt{1-b^{2} u_{S}^{2}}+\sqrt{1-b^{2} u_{R}^{2}}\right]+O\left(\frac{M^{2}}{b^{2}}, \frac{a M^{2}}{b^{3}}\right) .
\end{aligned}
$$


It follows that $a^{2}$ terms do not exist in this calculation.

By combining Eqs. (37) and (56), we obtain

$$
\begin{aligned}
-\iint_{\mathcal{C}_{R} \square} K d S-\int_{R}^{S} \kappa_{g} d \ell= & \frac{2 M}{b}\left(\sqrt{1-b^{2} u_{S}^{2}}+\sqrt{1-b^{2} u_{R}^{2}}\right) \\
& -\frac{2 a M}{b^{2}}\left(\sqrt{1-b^{2} u_{R}^{2}}+\sqrt{1-b^{2} u_{S}^{2}}\right) \\
& +O\left(\frac{M^{2}}{b^{2}}\right) .
\end{aligned}
$$

This equals to the right-hand side of Eq. (44). This means that the present approach is consistent with the Gauss-Bonnet theorem.

\section{CONCLUSION}

By using the Gauss-Bonnet theorem in differential geometry, we discussed a possible extension of the method of calculating the bending angle of light to stationary, axisymmetric and asymptotically flat spacetimes. We introduced a spatial metric $\gamma_{i j}$ to define the bending angle of light, which was shown to be coordinate-invariant.

We considered the light rays on the equatorial plane in the axisymmetric spacetime. We showed that the geodesic curvature of the photon orbit with $\gamma_{i j}$ can be nonzero in gravitomagnetism, even though the light ray in the four-dimensional spacetime follows the null geodesic. Finally, we considered Kerr spacetime in order to examine how the bending angle of light is computed by the present method. We made an order-of-magnitude estimate

of the finite-distance corrections for two possible astronomical cases; (1) the Sun and (2) the Sgr A*. The results suggest that the finite-distance corrections due to gravitomagnetism are unlikely to be observed with present technology.

However, our analysis on possible astronomical observations in this paper is limited within the Kerr model. It might be interesting to examine the gravitomagnetic bending of light by using other axisymmetric spacetimes in GR or in a specific theory of modified gravity. A further study along this direction is left for future.

\section{Acknowledgments}

We are grateful to Marcus Werner for the stimulating discussions, especially for his useful comments and his talk on the osculating Riemann approach at the seminar in Hirosaki 
university. We would like to thank Yuuiti Sendouda, Ryuichi Takahashi, Yuya Nakamura and Naoki Tsukamoto for the useful conversations. This work was supported in part by Japan Society for the Promotion of Science Grant-in-Aid for Scientific Research, No. 26400262 (H.A.), No. 17K05431 (H.A.) and in part by by Ministry of Education, Culture, Sports, Science, and Technology, No. 15 H00772 (H.A.) and No. 17H06359 (H.A.).

\section{Appendix A: Detailed calculations at $O\left(M^{2} / b^{2}\right)$ and $O\left(a^{2} / b^{2}\right)$}

First, we investigate $K$. Up to the second order, it is expanded as

$$
\begin{aligned}
K & =\frac{R_{r \phi r \phi}}{\gamma} \\
& =-\frac{2 M}{r^{3}}+\frac{3 M^{2}}{r^{4}}+O\left(\frac{a^{2} M}{r^{5}}\right),
\end{aligned}
$$

where $\gamma$ denotes $\operatorname{det}\left(\gamma_{i j}\right)$. Note that there are no $a^{2}$ terms in $K$. More interestingly, only the $a^{2} M$ term among the third order terms do exist in $K$. By noting that $K$ begins with $O(M)$, what we need for the second-order calculations is only the linear order in the area element on the equatorial plane. This is obtained as

$$
\begin{aligned}
d S & \equiv \sqrt{\gamma} d r d \phi \\
& =\left[r+3 M+O\left(\frac{M^{2}}{r}\right)\right] d r d \phi,
\end{aligned}
$$

where terms at $O(a)$ and also at $O\left(a^{2}\right)$ do not exist in $d S$. This is because all terms including the spin parameter cancel out in $\gamma$ for $\theta=\pi / 2$ and $\gamma$ thus depends only on $M$, as can be shown by direct calculations.

By using Eqs. (A1) and (A2), the surface integration of the Gaussian curvature is done 
as

$$
\begin{aligned}
& -\iint K d S=\int_{\infty}^{r_{O E}} d r \int_{\phi_{S}}^{\phi_{R}} d \phi\left(-\frac{2 M}{r^{3}}+\frac{3 M^{2}}{r^{4}}\right)(r+3 M)+O\left(\frac{M^{3}}{b^{3}}, \frac{a M^{2}}{b^{3}}, \frac{a^{2} M}{b^{3}}\right) \\
& =\int_{0}^{\frac{1}{b} \sin \phi+\frac{M}{b^{2}}\left(1+\cos ^{2} \phi\right)} d u \int_{\phi_{S}}^{\phi_{R}} d \phi\left(2 M+3 u M^{2}\right)+O\left(\frac{M^{3}}{b^{3}}, \frac{a M^{2}}{b^{3}}, \frac{a^{2} M}{b^{3}}\right) \\
& =\int_{\phi_{S}}^{\phi_{R}}\left[2 u M+\frac{3 u^{2}}{2} M^{2}\right]_{0}^{\frac{1}{b} \sin \phi+\frac{M}{b^{2}}\left(1+\cos ^{2} \phi\right)} d \phi+O\left(\frac{M^{3}}{b^{3}}, \frac{a M^{2}}{b^{3}}, \frac{a^{2} M}{b^{3}}\right) \\
& =\int_{\phi_{S}}^{\phi_{R}}\left[\frac{2 M}{b} \sin \phi+\frac{M^{2}}{2 b^{2}}\left(7+\cos ^{2} \phi\right)\right] d \phi+O\left(\frac{M^{3}}{b^{3}}, \frac{a M^{2}}{b^{3}}, \frac{a^{2} M}{b^{3}}\right) \\
& =\frac{2 M}{b}[\cos \phi]_{\phi_{R}}^{\phi_{S}}+\frac{M^{2}}{2 b^{2}}\left[\frac{30 \phi+\sin (2 \phi)}{4}\right]_{\phi_{S}}^{\phi_{R}}+O\left(\frac{M^{3}}{b^{3}}, \frac{a M^{2}}{b^{3}}, \frac{a^{2} M}{b^{3}}\right) \\
& =\frac{2 M}{b}\left[\sqrt{1-b^{2} u_{S^{2}}}+\sqrt{1-b^{2} u_{R}^{2}}\right] \\
& +\frac{2 M^{2}}{b}\left[\frac{u_{S}\left(2-b^{2} u_{S}^{2}\right)}{\sqrt{1-b^{2} u_{S}^{2}}}+\frac{u_{R}\left(2-b^{2} u_{R}^{2}\right)}{\sqrt{1-b^{2} u_{R}^{2}}}\right] \\
& +\frac{15 M^{2}}{4 b^{2}}\left[\pi-\arcsin \left(b u_{S}\right)-\arcsin \left(b u_{R}\right)\right] \\
& -\frac{M^{2}}{4 b^{2}}\left[b u_{S} \sqrt{1-b^{2} u_{S}^{2}}+b u_{R} \sqrt{1-b^{2} u_{R}^{2}}\right]+O\left(\frac{M^{3}}{b^{3}}, \frac{a M^{2}}{b^{3}}, \frac{a^{2} M}{b^{3}}\right) \\
& =\frac{2 M}{b}\left[\sqrt{1-b^{2} u_{S}^{2}}+\sqrt{1-b^{2} u_{R}^{2}}\right] \\
& +\frac{15 M^{2}}{4 b^{2}}\left[\pi-\arcsin \left(b u_{S}\right)-\arcsin \left(b u_{R}\right)\right] \\
& +\frac{M^{2}}{4 b^{2}}\left[\frac{b u_{S}\left(15-7 b^{2} u_{S}^{2}\right)}{\sqrt{1-b^{2} u_{S}^{2}}}+\frac{b u_{R}\left(15-7 b^{2} u_{R}^{2}\right)}{\sqrt{1-b^{2} u_{R}^{2}}}\right]+O\left(\frac{M^{3}}{b^{3}}, \frac{a M^{2}}{b^{3}}, \frac{a^{2} M}{b^{3}}\right),
\end{aligned}
$$

where we use, in the second line, an iterative solution for the orbit equation by Eq. (21) for the Kerr spacetime.

Next, we study the geodesic curvature. On the equatorial plane, we obtain

$$
\begin{aligned}
\kappa_{g} & =-\frac{1}{\sqrt{\frac{\Sigma^{2}}{\Delta(\Sigma-2 M r)}\left(r^{2}+a^{2}+\frac{2 a^{2} M r \sin ^{2} \theta}{\Sigma}\right) \frac{\Sigma \sin ^{2} \theta}{(\Sigma-2 M r)}}} \beta_{\phi, r} \\
& =-\frac{2 a M}{r^{3}}+O\left(\frac{a M^{2}}{r^{3}}\right)
\end{aligned}
$$


where $a^{2}$ terms do not exist. From this, we obtain

$$
\begin{aligned}
\int_{c_{p}} \kappa_{g} d \ell & =-\int_{S}^{R} d \ell\left[\frac{2 a M}{r^{2}}+O\left(\frac{a M^{2}}{r^{3}}\right)\right] \\
& =-\frac{2 a M}{b^{2}} \int_{\phi_{S}}^{\phi_{R}} \cos \vartheta d \vartheta+O\left(\frac{a M^{2}}{b^{3}}\right) \\
& =-\frac{2 a M}{b^{2}}\left[\sin \phi_{R}-\sin \phi_{S}\right]+O\left(\frac{a M^{2}}{b^{3}}\right) \\
& =\frac{2 a M}{b^{2}}\left[\sqrt{1-b^{2} u_{R}^{2}}+\sqrt{1-b^{2} u_{S}^{2}}\right]+O\left(\frac{a M^{2}}{b^{3}}\right),
\end{aligned}
$$

where we use $\sin \phi_{S}=\sqrt{r_{S}^{2}-b^{2}} / r_{S}+O\left(M / r_{S}\right)$ and $\sin \phi_{R}=-\sqrt{r_{R}^{2}-b^{2}} / r_{R}+O\left(M / r_{R}\right)$.

By combining Eqs. ( $\mathrm{A} 3$ ) and ( $(\mathrm{A} 5)$, we obtain

$$
\begin{aligned}
\alpha \equiv & -\iint_{\substack{\infty \\
\square_{S}}} K d S-\int_{R}^{S} \kappa_{g} d l \\
= & \frac{2 M}{b}\left[\sqrt{1-b^{2} u_{S}^{2}}+\sqrt{1-b^{2} u_{R}^{2}}\right] \\
& +\frac{15 M^{2}}{4 b^{2}}\left[\pi-\arcsin \left(b u_{S}\right)-\arcsin \left(b u_{R}\right)\right] \\
& +\frac{M^{2}}{4 b^{2}}\left[\frac{b u_{S}\left(15-7 b^{2} u_{S}^{2}\right)}{\sqrt{1-b^{2} u_{S}^{2}}}+\frac{b u_{R}\left(15-7 b^{2} u_{R}^{2}\right)}{\sqrt{1-b^{2} u_{R}^{2}}}\right] \\
& -\frac{2 a M}{b^{2}}\left[\sqrt{1-b^{2} u_{R}^{2}}+\sqrt{1-b^{2} u_{S}^{2}}\right]+O\left(\frac{M^{3}}{b^{3}}, \frac{a M^{2}}{b^{3}}, \frac{a^{2} M}{b^{3}}\right) .
\end{aligned}
$$

Note that $a^{2}$ terms and $a^{3}$ ones do not appear in $\alpha$ for the finite distance situation as well as in the infinite distance limit. If we assume the infinite distance limit $u_{R}, u_{S} \rightarrow 0$, Eq. (A6 ) becomes

$$
\alpha \rightarrow \frac{4 M}{b}+\frac{15 \pi M^{2}}{4 b^{2}}-\frac{4 a M}{b^{2}} .
$$

This agrees with the previous results, especially the numerical coefficients at the order of $M^{2}$ and $a M$.

[1] A. Einstein, Ann. Phys. (Berlin) 49, 769 (1916).

[2] F. W. Dyson, A. S. Eddington, C. Davidson, Phil. Trans. R. Soc. A 220, 291 (1920).

[3] Y. Hagihara, Jpn. J Astron. Geophys. 8, 67 (1931).

[4] S. Chandrasekhar, The Mathematical Theory of Black Holes, (Oxford University Press, New York, 1998). 
[5] C. W. Misner, K. S. Thorne, J. A. Wheeler, Gravitation, (Freeman, New York, 1973).

[6] C. Darwin, Proc. R. Soc. A 249, 180 (1959).

[7] V. Bozza, Phys. Rev. D 66, 103001 (2002).

[8] S. V. Iyer and A. O. Petters, Gen. Relativ. Gravit. 39, 1563 (2007).

[9] V. Bozza, and G. Scarpetta, Phys. Rev. D 76, 083008 (2007).

[10] S. Frittelli, T. P. Kling, and E. T. Newman, Phys. Rev. D 61, 064021 (2000).

[11] K. S. Virbhadra, and G. F. R. Ellis, Phys. Rev. D 62, 084003 (2000).

[12] K. S. Virbhadra, Phys. Rev. D 79, 083004 (2009).

[13] K. S. Virbhadra, D. Narasimha, and S. M. Chitre, Astron. Astrophys. 337, 1 (1998).

[14] K. S. Virbhadra, and G. F. R. Ellis, Phys. Rev. D 65, 103004 (2002).

[15] K. S. Virbhadra, and C. R. Keeton, Phys. Rev. D 77, 124014 (2008).

[16] S. Zschocke, Class. Quantum Grav. 28, 125016 (2011).

[17] E. F. Eiroa, G. E. Romero, and D. F. Torres, Phys. Rev. D 66, 024010 (2002).

[18] V. Perlick, Phys. Rev. D 69, 064017 (2004).

[19] F. Abe, Astrophys. J. 725, 787 (2010).

[20] Y. Toki, T. Kitamura, H. Asada, and F. Abe, Astrophys. J. 740, 121 (2011).

[21] K. Nakajima, and H. Asada, Phys. Rev. D 85, 107501 (2012).

[22] G. W. Gibbons, and M. Vyska, Class. Quant. Grav. 29065016 (2012).

[23] J. P. DeAndrea, and K. M. Alexander, Phys. Rev. D 89, 123012 (2014).

[24] T. Kitamura, K. Nakajima, and H. Asada, Phys. Rev. D 87, 027501 (2013).

[25] N. Tsukamoto, and T. Harada, Phys. Rev. D 87, 024024 (2013).

[26] K. Izumi, C. Hagiwara, K. Nakajima, T. Kitamura, and H. Asada, Phys. Rev. D 88, 024049 (2013).

[27] T. Kitamura, K. Izumi, K. Nakajima, C. Hagiwara, and H. Asada, Phys. Rev. D 89, 084020 (2014).

[28] K. Nakajima, K. Izumi, and H. Asada, Phys. Rev. D 90, 084026 (2014).

[29] N. Tsukamoto, T. Kitamura, K. Nakajima, and H. Asada, Phys. Rev. D 90, 064043 (2014).

[30] G. W. Gibbons, and M. C. Werner, Class. Quant. Grav. 25, 235009 (2008).

[31] A. Ishihara, Y. Suzuki, T. Ono, T. Kitamura, and Hideki Asada, Phys. Rev. D 94, 084015 (2016).

[32] A. Ishihara, Y. Suzuki, T. Ono, and Hideki Asada, Phys. Rev. D 95, 044017 (2017). 
[33] R. Epstein, and I. I. Shapiro, Phys. Rev. D 22, 2947 (1980).

[34] J. Ibanez, Astron. Astrophys. 124, 175 (1983).

[35] H. Asada, and M. Kasai, Prog. Theor. Phys. 104, 95 (2000).

[36] S. Kopeikin, and B. Mashhoon, Phys. Rev. D 65, 064025 (2002).

[37] M. C. Werner, Gen. Rel. Grav. 44, 3047 (2012).

[38] In a conventional approach, precise analytic treatments of the deflection angle of light were done in the equatorial plane of a Kerr black hole [39] and for generic photon orbits in terms of the generalized hypergeometric functions of Appell and Lauricella [40], though their calculations are entirely based on an assumption that both the source and the receiver are located at the null infinity.

[39] S. V. Iyer, and E. C. Hansen, Phys. Rev. D 80, 124023 (2009).

[40] G. V. Kraniotis, Class. Quant. Grav. 28, 085021 (2011).

[41] M. P. Do Carmo, Differential Geometry of Curves and Surfaces, pages 268-269, (Prentice-Hall, New Jersey, 1976).

[42] T. Lewis, Proc. Roy. Soc. A, 136, 176 (1932).

[43] H. Levy, and W. J. Robinson, Proc. Camb. Phil. Soc. 60, 279 (1963).

[44] A. Papapetrou, Ann. Inst. H. Poincare A, 4, 83 (1966).

[45] In this paper, we use the polar coordinates. In the cylindrical coordinates, the line element is known as the Weyl-Lewis-Papapetrou form [42 44]].

[46] In this section and after, we do not call $\gamma_{i j}$ the optical metric, because the photon orbit is not always a geodesic in a space defined by $\gamma_{i j}$. See Eq. (9).

[47] A. C. Belton, Geometry of Curves and Surfaces, page 38 (2015); www.maths.lancs.ac.uk/ belton/www/notes/geom_notes.pdf; J. Oprea, Differential Geometry and Its Applications (2nd Edition), page 210, (Prentice Hall, New Jersey, 2003).

[48] F. P. Pijpers, Mon. Not. Roy. Astron. Soc. 297, L76 (1998); S. L. Bi, T. D. Li, L. H. Li, and W. M. Yang, Astrophys. J. Lett. 731, L42 (2011).

[49] http://sci.esa.int/gaia/

[50] http://www.jasmine-galaxy.org/index-en.html 


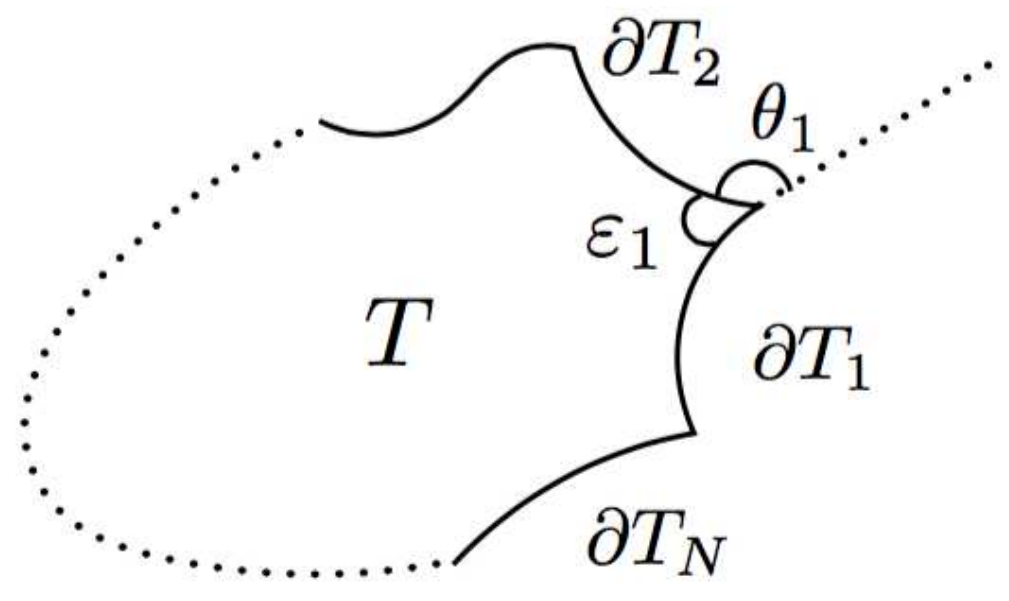

FIG. 1: Schematic figure for the Gauss-Bonnet theorem.

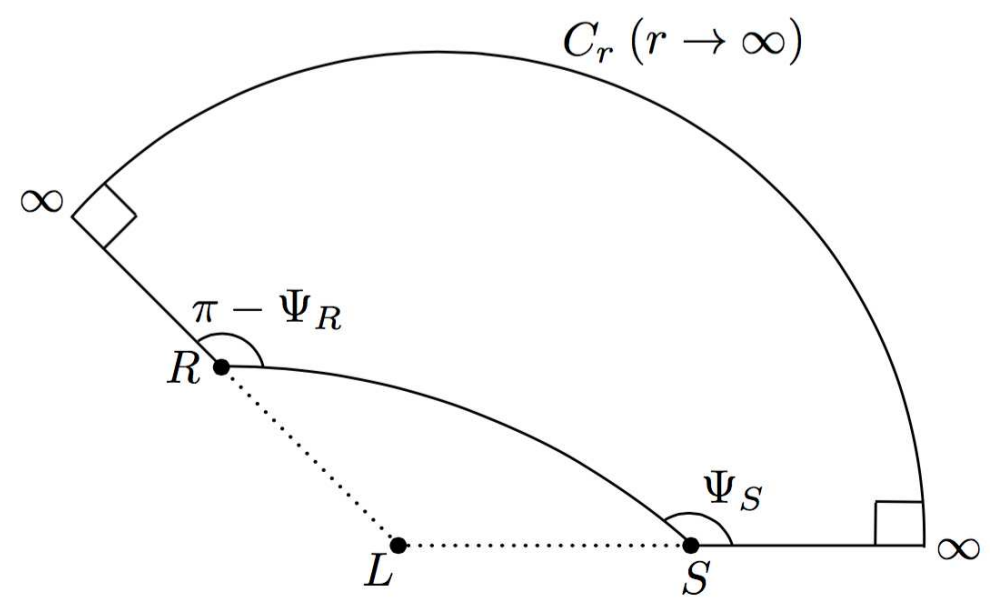

FIG. 2: Quadrilateral ${ }_{R}^{\infty} \square_{S}^{\infty}$ embedded in a curved space. Note that the inner angle at the vertex $R$ is $\pi-\Psi_{R}$. 


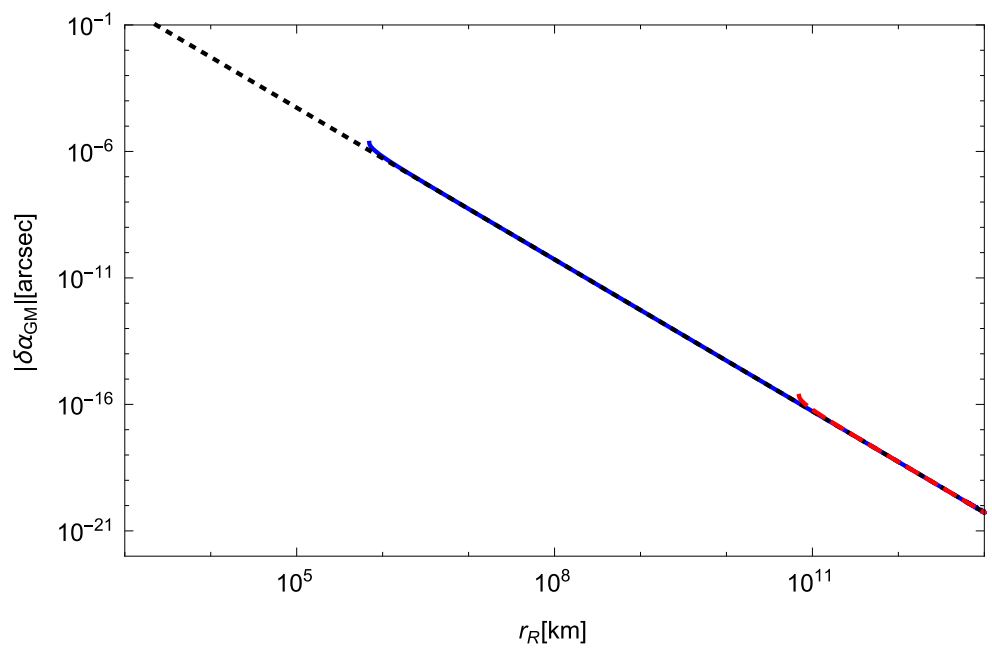

FIG. 3: $\delta \alpha_{G M}$ for the Sun. The vertical axis denotes the finite-distance correction to the gravitomagnetic deflection angle of light and the horizontal axis denotes the receiver distance $r_{R}$. The solid curve (blue in color) and dashed one (red in color) correspond to $b=R_{\odot}$ and $b=10 R_{\odot}$, respectively. The dotted line (black in color) denotes the leading term of $\delta \alpha_{G M}$ given by Eq. (49). The overlap between these curves suggest that the dependence of $\delta \alpha_{G M}$ on the impact parameter $b$ is very weak.

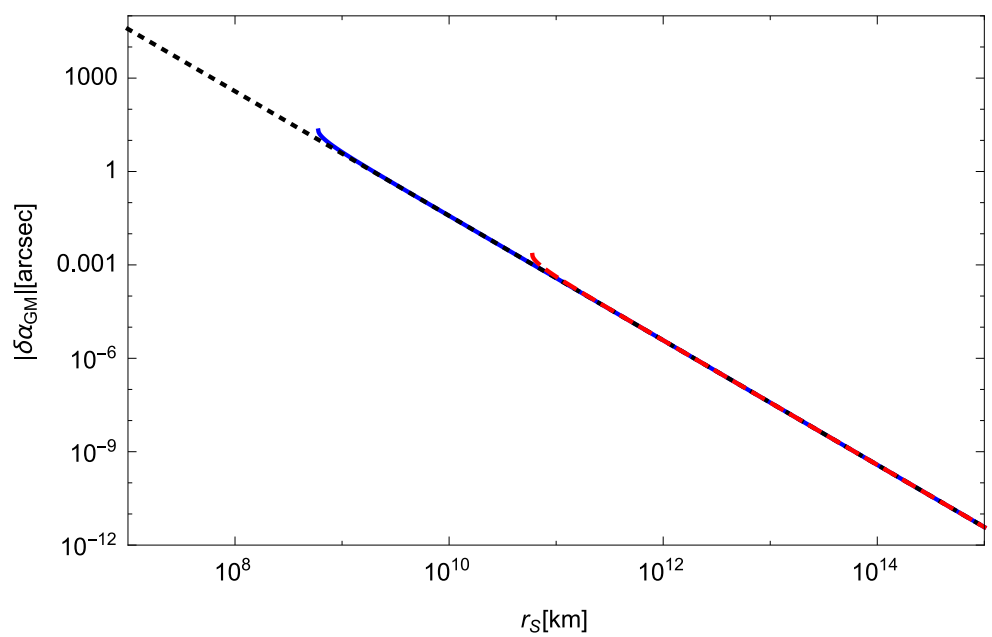

FIG. 4: $\delta \alpha_{G M}$ for the Sgr A*. The vertical axis denotes the finite-distance correction to the deflection angle of light and the horizontal axis denotes the source distance $r_{S}$. The solid curve (blue in color) and dashed one (red in color) correspond to $b=10^{2} M$ and $b=10^{4} M$, respectively. The dotted line (yellow in color) denotes the leading term of $\delta \alpha_{G M}$ given by Eq. (49). The overlap between these plots suggest that $\delta \alpha_{G M}$ depends faintly on the impact parameter $b$. 